\title{
EFEITO DAS CONDIÇÕES DE MANEJO NAS CARACTERÍSTICAS SENSORIAIS DE BANANA (Musa spp.) cv. PACOVAN ${ }^{1}$ \\ MARIA SUELI FRANCISCO ${ }^{2}$, RAUNIRA DA COSTA ARAÚJO 3 , ESMERALDA PARANHOS DOS SANTOS ${ }^{4}$, FRANCISCO FERNANDES BATISTA GOMES ${ }^{5}$, GEORGE RODRIGO BELTRÃO DA CRUZ ${ }^{6}$
}

RESUMO - Estudou-se o efeito de quatro condições de manejo sobre a aceitação de banana 'Pacovan'. Os frutos foram colhidos no ponto de maturação fisiológica ideal para o armazenamento, em quatro propriedades rurais do município de Bananeiras - PB, seguindo-se delineamento inteiramente casualizado. Os tratamentos foram identificados como: $\mathbf{P}_{1}$ : irrigação+adubação química e orgânica +controle químico de plantas invasoras; $\mathbf{P}_{2}$ : controle manual de plantas invasoras; $\mathbf{P}_{3}$ : irrigação+adubação química+controle manual de plantas invasoras; $\mathbf{P}_{4}$ : controle manual e químico de plantas invasoras. Em cada propriedade, foram colhidos cinco cachos de banana que foram submetidos à climatização com carbureto de cálcio por 12 horas para uniformização da maturação. Após esse período, foram mantidos à temperatura ambiente até sua completa maturação. O painel sensorial foi composto por 62 julgadores não treinados, com idade média de 18 anos. Os atributos aroma, sabor, textura e cor da polpa foram avaliados por meio de teste de aceitação, utilizando-se de escala hedônica estruturada de 9 pontos. Para o teste de intenção de compra, foi utilizada escala estruturada de 5 pontos. As amostras foram apresentadas aos julgadores na forma de rodelas, com espessura aproximada de $1,5 \mathrm{~cm}$. Os resultados foram submetidos à análise de variância (ANOVA), e as médias, comparadas pelo teste de Tukey, ao nível de $5 \%$ de probabilidade. Para todos os atributos avaliados,os valores situaram-se entre 7,34 e 7,68, posicionando-se na escala hedônica entre os termos 'gostei moderadamente' e 'gostei muito'. Para intenção de compra, o percentual de aceitação foi de 74,19; 72,58; 72,58 e 70,97\%, respectivamente, para as amostras $\mathrm{P}_{1}, \mathrm{P}_{2}, \mathrm{P}_{3}$ e $\mathrm{P}_{4}$. O tipo de manejo não influenciou na aceitação da banana 'Pacovan', sendo os frutos considerados pelos julgadores como produto de boa aceitação.

Termos para Indexação: manejo, atributos, escala hedônica, julgadores.

\section{EFECT OF MANAGEMENT CONDITIONS ON THE SENSORY CHARACTERISTICS OF BANANA (Musa spp.) cv. PACOVAN}

\begin{abstract}
The effect of four management conditions on the acceptance of 'Pacovan' banana was assessed. The fruits were harvested at ideal physiological maturity for storage in four farms in the municipality of Bananeiras - PB, according to a fully randomized design. Treatments were identified as P1: Irrigation + chemical and organic fertilization + chemical control of weeds; P2: manual control of weeds; P3: irrigation + chemical fertilization + manual control of weeds; P4: manual and chemical control of weeds. Five bunches of bananas were harvested in each farm and submitted to cooling with calcium carbide for 12 hours for uniform ripening. After this period, fruits were kept at room temperature until full maturation. The sensory panel was composed of 62 untrained judges, with mean age of 18 years. Attributes of aroma, flavor, texture and pulp color were assessed through acceptance test using a 9-point hedonic scale. The purchase intention test used a 5-point structured scale. The samples were presented to panelists in the form of slices with thickness of approximately $1.5 \mathrm{~cm}$. Results were submitted to analysis of variance (ANOVA) and means were compared by the Tukey test at 5\% probability. For all attributes, values ranged from 7.34 to 7.68 , in the hedonic scale between terms 'liked moderately' and 'liked very much'. As for the purchase intention, acceptance percentages were 74.19, 72.58, 72.58 and 70.97\% respectively for P1, P2, P3 and P4. The management condition did not influence the acceptance of 'Pacovan' banana, and the fruits were considered by the judges as a product of good acceptance.
\end{abstract}

Index terms: management, attributes, hedonic scale, judges.

\footnotetext{
${ }^{1}$ (Trabalho 111-13). Recebido em: 13-03-2013. Aceito para publicação em: 25-03-2014. Parte da dissertação de mestrado do primeiro autor. Projeto financiado pelo CNPq ((Processo n $\left.{ }^{\circ} 478400 / 2008-0\right)$.

${ }_{2}^{2}$ Bacharel em Agroindústria, pela Universidade Federal da Paraíba, Campus III. E-mail: suelipb.agro@yahoo.com.br

${ }^{3}$ Eng. Agrônoma, Dra ${ }^{2}$ Professora da Universidade Federal da Paraíba, Campus III. E-mail: arinuar@hotmail.com

${ }^{4}$ Eng. de Alimentos, Dra . Professora da Universidade Federal da Paraíba, Campus I. E-mail: esmeraldaparanhos@ig.com.br

${ }^{5}$ Técnico Agrícola, funcionário da Empresa de Assistência Técnica e Extensão Rural da Paraíba. E-mail: batista-gomes-@hotmail.com

${ }^{6}$ Eng. Agrônomo, Dr. Professor da Universidade Federal da Paraíba, Campus III. E-mail: georgebeltrao@hotmail.com
} 


\section{INTRODUÇÃO}

As bananeiras (Musa spp.) produtoras de frutos comestíveis pertencem à classe das Monocotiledôneas, ordem Scitaminales, família Musaceae, subfamília Musoideae, gênero Musa e seções Eumusa e Australimusa. Na seção Eumusa, localizam-se as bananas comestíveis, de grande valor comercial (MANICA, 1998), originária do continente Asiático, de onde foi disseminada para várias partes do mundo. No Brasil, a cultura encontrou boas condições de desenvolvimento, sendo cultivada em todas as regiões; entretanto, em alguns estados, o cultivo sofre restrições em virtude de fatores climáticos. Em 2012, a produção de banana no Brasil foi de 6.846.611 t, com rendimento médio 14.361 $\mathrm{kg} / \mathrm{ha}$. Para safra de 2013, estima-se 7.303.967 t (IBGE, 2013).

Segundo Silva et al. (1999), no Brasil, existe um número expressivo de variedades de banana; no entanto, quando se consideram aspectos como preferência dos consumidores, produtividade, tolerância a pragas e doenças, resistência à seca, porte e resistência ao frio, são poucas as cultivares com potencial agronômico para a exploração comercial. Embora a cultivar Pacovan não agrupe todas essas qualidades, figura no cenário nacional como uma das mais difundidas, sendo uma das cultivares mais rústicas (SILVA et al., 2002). Em virtude de sua rusticidade tornou-se uma das melhores opções para os produtores, principalmente das regiões Norte e Nordeste do País, onde o cultivo é conduzido com adoção de baixa tecnologia. Outro fator que contribuiu para a cultivar ser uma das mais plantadas é a boa aceitação dos consumidores. Matsuura et al. (2004) relatam que a escolha da variedade pelo produtor é consequência, entre outros fatores, de alguns atributos de qualidade dos frutos, tais como: sabor, vida útil e aparência, características fundamentais ao consumidor e que afetam sua decisão de compra. Assim, de nada adiantaria a cultivar apresentar boas características agronômicas se fossem rejeitadas pelos consumidores.

A banana atrai seus consumidores pelas suas propriedades nutricionais e aspectos sensoriais, além de sua praticidade de consumo, podendo ser consumida in natura ou processada. A casca da banana constitui-se em uma 'embalagem' individual, de fácil remoção, higiênica e prática. A ausência de suco na polpa, de sementes duras e sua disponibilidade durante todo o ano também contribuem para sua aceitação (LICHTEMBERG, 1999).

De acordo com Matsuura et al. (2002), a análise sensorial de frutos das cultivares e híbridos de plantas deve ser realizada a fim de se verificar a aceitação dos mesmos por parte dos consumidores. Minin (2010) relata que, por meio de teste de aceitação, os consumidores expressam o quanto gostam ou desgostam de um produto.

O objetivo da pesquisa foi avaliar o efeito de quatro condições de manejo sobre a aceitação de frutos de banana 'Pacovan'.

\section{MATERIAL E MÉTODOS}

O trabalho foi desenvolvido no Centro de Ciências Humanas Sociais e Agrárias (CCHA) da Universidade Federal da Paraíba (UFPB), entre os meses de fevereiro e abril de 2011. A seleção das amostras foi realizada segundo as condições de manejo do bananal, quanto à irrigação, adubação química, adubação orgânica e controle químico ou manual de plantas invasoras. Os tratamentos consistiram em coletas de cinco cachos de banana da cultivar Pacovan, em quatro propriedades rurais localizadas no município de Bananeiras - PB. Os tratamentos foram identificados como: $\mathbf{P}_{1}$ : irrigação+adubação química e orgânica +controle químico de plantas invasoras; $\mathbf{P}_{\mathbf{2}}$ : controle manual de plantas invasoras; $\mathbf{P}_{3}$ : irrigação+adubação química+controle manual de plantas invasoras; $\mathbf{P}_{\mathbf{4}}$ : controle manual e químico de plantas invasoras.

\section{Características de manejo dos bananais}

P1 - o manejo adotado não segue as recomendações técnicas adotadas para a cultura. A irrigação consiste em deixar a água espalhar-se por gravidade pelas fileiras do plantio até ocorrer a inundação e penetração da água no solo em uma grande área. Quando isso ocorre, a água passa a ser desviada para outra área, sempre obedecendo a um rodízio durante todo o período de seca. A adubação, tanto a química quanto a orgânica (esterco bovino), é realizada sem conhecimento das exigências nutricionais do plantio, já que nunca foi realizada análise de solo e de folhas. Esse é um plantio com cerca de cinco anos de idade, e o controle de plantas invasoras é realizado sempre que necessário, por meio de herbicida.

P2 - esse bananal caracteriza-se por apresentar características orgânicas. É um plantio com mais de 15 anos, sendo conduzido com muitos perfilhos por touceira. $\mathrm{O}$ único trato cultural adotado é o controle manual de plantas invasoras sempre que necessário.

P3 - plantio no primeiro ciclo de produção, e o manejo adotado segue as recomendações técnicas para a cultura quanto à irrigação (por gotejamento), 
adubação química e controle de plantas invasoras.

P4 - plantio com mais de 16 anos de idade, conduzido com muitos perfilhos por touceira, e não segue as técnicas recomendadas para a cultura. $\mathrm{O}$ controle de plantas invasoras é realizado sempre que há necessidade, por meio da aplicação de herbicida e manual, predominando o segundo.

Os frutos foram colhidos no ponto de maturação fisiológica (identificado visualmente, levando-se em consideração as observações dos produtores, que consideram a ausência ou redução das quinas ou angulosidades da superfície dos frutos como indicativo do momento ideal para colheita), transportados para o Laboratório de Biologia do CCHSA, onde foram identificados, lavados para a remoção dos restos florais e do látex, e secos ao ar livre. Após a higienização, foram selecionados os três frutos centrais da segunda penca de cada cacho e submetidos à determinação dos Sólidos Solúveis Totais (SST). A leitura foi feita por refratometria, utilizando-se do refratômetro modelo RT 3ATC, marca NOVA. Para padronização, somente os cachos que tinham frutos com teor de SST entre 4,80\% e $4,88 \%$ foram aceitos como amostras, sendo este estágio de maturação codificado como verde. Em seguida, os frutos foram submetidos à climatização com carbureto de cálcio, por 12 horas, para a uniformização da maturação. Após esse período, os frutos foram mantidos à temperatura ambiente até sua completa maturação, o que ocorreu quando a coloração da casca estava completamente amarela, o que corresponde ao estágio 6 na escala de maturação de VON LOESECKE (CEAGESP, 2006), e a polpa apresentava ausência de sabor adstringente, sendo este estágio codificado como maduro. Três frutos centrais da segunda penca de cada cacho no estágio maduro foram submetidos à análise dos SSTs.

A análise sensorial foi realizada no Laboratório de Análise Sensorial do CCHSA/UFPB em cabines individuais, com iluminação adequada e longe de ruídos e odores. O painel sensorial foi composto por 62 julgadores não treinados, selecionados entre a comunidade do CCHSA/UFPB, com média de idade de 18 anos, todos consumidores de banana. Empregou-se o teste de aceitação, utilizando-se de escala hedônica estruturada de nove pontos (9 = gostei muitíssimo; 1= desgostei muitíssimo) (MININ, 2010). As amostras foram apresentadas aos julgadores à temperatura ambiente, servidas na forma de rodelas com espessura aproximada de 1,5 $\mathrm{cm}$, em copos descartáveis transparentes, codificados com números de três dígitos, definidos de forma aleatória. Foram avaliados quanto aos atributos: aroma, sabor, textura e cor da polpa. Também foi aplicado o teste de intenção de compra, utilizando-se de escala estruturada de cinco pontos $(5=$ certamente eu compraria; 1 = certamente eu não compraria).

Os dados foram analisados como delineamento inteiramente casualizado, considerando-se o tipo de manejo como fator de variação. Os resultados foram submetidos à análise de variância (ANOVA); e as médias, comparadas pelo teste de Tukey, ao nível de $5 \%$ de probabilidade.

\section{RESULTADOS E DISCUSSÃO}

Os valores médios de Sólidos Solúveis Totais (SST) para as amostras coletadas nas quatro áreas, tanto para os frutos verdes como para os maduros analisados, podem ser observados na Tabela 1. Os valores não diferiram entre si no estádio verde, mostrando que se conseguiu a padronização inicial buscada. Percebe-se que o tipo de manejo não influenciou significativamente os SSTs no estágio maduro da banana 'Pacovan', que variaram entre $21,61 \%$ e $22,50 \%$.

$\mathrm{O}$ aumento dos SSTs era esperado devido às reações bioquímicas que ocorrem durante $\mathrm{o}$ processo de amadurecimento das bananas. Durante o amadurecimento, ocorre a transformação do amido, principal constituinte do fruto, em açúcares solúveis. Em frutos maduros da cultivar Pacovan, a porcentagem de SST oscila entre $17,5 \%$ e $28,3 \%$ (CERQUEIRA et al., 2002; MATSUURA et al., 2002; JESUS et al., 2004; SALLES et al., 2006; LEITE et al., 2010). Estes autores estudaram frutos no mesmo estágio de maturação, avaliados nesta pesquisa (estágio 6). Fatores como manejo, condições edafoclimáticas na qual o fruto foi produzido e até mesmo a época de colheita podem determinar diferenças entre os SSTs para a mesma cultivar.

Os teores de sólidos solúveis são importantes na determinação da qualidade e da aceitação da fruta por ser um indicador do teor de açúcares, consequentemente, um indicador do grau de doçura. Matsuura et al. (2004), estudando preferências do consumidor de um mercado local em Cruz das Almas - BA, considerando os atributos de qualidade dos frutos frescos de banana madura, observaram que $61,6 \%$ dos entrevistados preferem frutos de banana com doçura média, 28,9\% frutos muito doces e $9,5 \%$ frutos pouco doces, confirmando a preferência do consumidor brasileiro por alimentos mais adocicados.

Os valores médios para a aceitação da banana 'Pacovan', utilizando escala hedônica, mostraram não existir diferença significativa $(\mathrm{p}>0,05)$ entre as amostras para nenhum dos atributos avaliados: 
aroma, sabor, textura e cor (Tabela 2). Para todos os atributos, os valores situaram-se entre 7,34 e 7,68 . Tais valores aproximam-se de 8 que, na escala hedônica estruturada de 9 pontos, corresponde a 'gostei muito', sendo, portanto, considerados como produto de boa aceitação. Os valores para todos os atributos avaliados, exceto para a cor, foram maiores que os encontrados por Matsuura et al. (2002) ao estudarem a aceitação de frutos da cultivar Pacovan, oriundos de experimentos seguindo as recomendações técnicas usuais para a cultura: aroma 6,5 ; sabor 7,0 ; textura 6,8 e cor 7,8 .

Observou-se que o sabor e a cor obtiveram os maiores valores para todas as amostras, com média geral de 7,58 e 7,57, respectivamente. Matsuura et al. (2002) também verificaram que estes foram os atributos mais aceitos, com valor 7,0 para o sabor e 7,8 para a cor. De acordo com estes autores, dentre o conjunto de atributos avaliados, o sabor e a cor são de fundamental importância, sendo que a cor, por estar ligada à atratividade para o consumidor, e o sabor, por se relacionar com a preferência pela variedade.

Matsuura et al. (2004) observaram, ainda, que além do sabor, a vida útil e a aparência dos frutos são os atributos mais importantes na escolha ou na compra dos frutos de banana. Silva et al. (2002) também mencionam que o sabor é um dos atributos relacionados ao fruto que mais influenciam na preferência do consumidor

Com relação à intenção de compra, todas as amostras foram bem aceitas. Em teste de intenção de compra, a aceitação de um produto corresponde às notas 4 (provavelmente compraria) e 5 (certamente eu compraria), e, neste trabalho, o percentual de aceitação nessa faixa foi de $74,2 \%, 72,6 \%, 72,6 \%$ e $70,1 \%$, respectivamente, para as amostras dos tratamentos $\mathrm{P}_{1}, \mathrm{P}_{2}, \mathrm{P}_{3}$ e $\mathrm{P}_{4}$. Todas as amostras podem ser consideradas como bem aceitas (Figura 1). Esta alta aceitação confirma o gosto do nordestino pelas bananas do subgrupo Prata, especialmente pela 'Pacovan', cultivar tradicionalmente plantada e consumida na região.

As amostras da banana 'Pacovan' avaliadas, coletadas em quatro áreas diferentes, onde variou o local, a idade das plantas, além das condições de cultivo e de manejo, apresentaram valores considerados normais para os SSTs e foram bem aceitas pelos julgadores. Observa-se que a amostra $\mathrm{P}_{2}$, cultivada com características orgânicas, apresentou resultados semelhantes às demais amostras, tanto para os atributos sensoriais, como para o teor de SST.

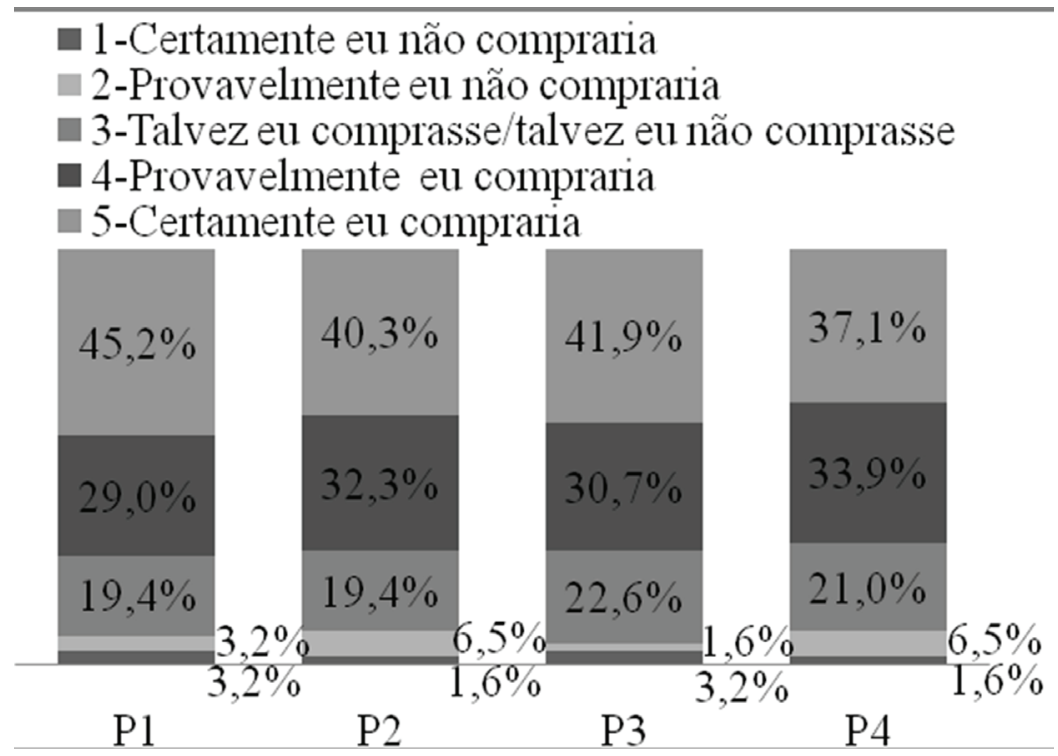

FIGURA 1 - Porcentagem das notas atribuídas pelos julgadores em relação à intenção de compra para banana 'Pacovan', em função do tipo de manejo. 
TABELA 1-Valores médios de Sólidos Solúveis Totais (SST) para polpa verde (V) e madura (M) dos frutos de banana 'Pacovan', em função do tipo de manejo.

\begin{tabular}{cccccc}
\hline \multirow{2}{*}{ SST (\%) } & \multicolumn{5}{c}{ Tratamentos } \\
\cline { 2 - 6 } & $\mathbf{P}_{1}$ & $\mathbf{P}_{2}$ & $\mathbf{P}_{3}$ & $\mathbf{P}_{4}$ & $\mathbf{C V ~ ( \% )}$ \\
\hline Verde & $4,8 \mathrm{a}$ & $4,9 \mathrm{a}$ & $4,8 \mathrm{a}$ & $4,8 \mathrm{a}$ & 1,9 \\
Madura & $22,5 \mathrm{a}$ & $22,3 \mathrm{a}$ & $21,6 \mathrm{a}$ & $22,5 \mathrm{a}$ & 3,9 \\
\hline
\end{tabular}

TABELA 2 -Valores médios para os atributos aroma, sabor, textura e cor da polpa dos frutos de banana 'Pacovan', em função do tipo de manejo.

\begin{tabular}{ccccc}
\hline \multirow{2}{*}{ Tratamentos } & \multicolumn{4}{c}{ Atributos } \\
\cline { 2 - 5 } & Aroma & Sabor & Textura & Cor \\
\hline $\mathbf{P}_{1}$ & $7,50 \mathrm{a}$ & $7,52 \mathrm{a}$ & $7,37 \mathrm{a}$ & $7,53 \mathrm{a}$ \\
$\mathbf{P}_{\mathbf{2}}$ & $7,43 \mathrm{a}$ & $7,68 \mathrm{a}$ & $7,47 \mathrm{a}$ & $7,63 \mathrm{a}$ \\
$\mathbf{P}_{\mathbf{3}}$ & $7,48 \mathrm{a}$ & $7,61 \mathrm{a}$ & $7,34 \mathrm{a}$ & $7,50 \mathrm{a}$ \\
$\mathbf{P}_{\mathbf{4}}$ & $7,50 \mathrm{a}$ & $7,53 \mathrm{a}$ & $7,52 \mathrm{a}$ & $7,61 \mathrm{a}$ \\
\hline $\mathbf{M G}$ & 7,48 & 7,58 & 7,42 & 7,57 \\
\hline$* \mathbf{C V}(\%)$ & 16,67 & 15,31 & 17,40 & 16,88 \\
\hline
\end{tabular}

\section{REFERÊNCIAS}

CERQUEIRA, R. C.; SILVA, S. O.; MEDINA, V. M. Características pós-colheita de frutos de genótipos de bananeira (Musa spp.). Revista Brasileira de Fruticultura, Jaboticabal, v. 24, n.3, p. 654-657, 2002.

CEAGESP - Centro de Qualidade em Horticultura. Normas de classificação de banana. São Paulo: CEAGESP/ PBMH \& PIF, 2006. (Documento, 29). Disponível em: <http://www.ceagesp.gov.br/ produtor/classific/>. Acesso em: 18 jul. 2013.

IBGE - Instituto Brasileiro de Geografa e Estatistica. Previsão de safra. Disponível em: $\leq$ www.sidra.ibge. gov.br>. Acesso em: 15 fev. 2013.

JESUS, S. C. et al. Caracterização física e química de frutos de diferentes genótipos de bananeira. Bragantia, Campinas, v.63, n.3, p. 315-323, 2004.

LEITE, G. A. et al. Qualidade pós-colheita da banana 'Pacovan' comercializada em diferentes estabelecimentos no município de Mossoró-RN. Revista Brasileira de Ciências Agrárias, Recife, v.5, n.3, p.322-327, 2010.

LICHTEMBERG, L. A. Colheita e pós-colheita da banana. Informe Agropecuário, Belo Horizonte, v.20, n.196, p.73-90, 1999.

MANICA, I. Bananas: do plantio ao amadurecimento. Porto Alegre: Cinco Continentes, 1998. p.99.
MATSUURA, F. C. A. U.; CARDOSO, R. L.; RIBEIRO, D. E. Qualidade Sensorial de Frutos de Híbridos de Bananeira Cultivar Pacovan. Revista Brasileira de Fruticultura, Jaboticabal, v. 24, n. 1, p. 263-266, 2002.

MATSUURA, F. C. A. U.; COSTA, J. I. P.; FOLEGATTI, M. I. S. Marketing de Banana: Preferências do Consumidor Quanto aos Atributos de Qualidade dos Frutos. Revista Brasileira de Fruticultura, Jaboticabal, v. 26, n. 1, p. 48-52, 2004.

MININ, V. P. R. Análise sensorial: estudos com consumidores. Viçosa, MG: Editora UFLA, 2010. p. 308 .

SALLES, J. R. J.; NETO, J. A. M.; GUSMÃO, L. L. Qualidade da banana "Pacovan" comercializada no período maio - Outubro de 2003 em São Luís MA. Revista da FZVA, Uruguaiana, v.13, n.2. p. 90-96, 2006. Disponível em: <http://caioba.pucrs. br/ojs/index.php/fzva/article/view/2367> . Acesso em: 1 dez. 2011.

SILVA, J. T. A.; BORGES, A. L.; MALBURG, J. L. Solos, adubação e nutrição da bananeira. Informe Agropecuário, Belo Horizonte, v. 20, n. 196, p. 21-36, 1999.

SILVA, S. O.; FLORES, J. C. O.; LIMA NETO, F. P. Avaliação de cultivares e híbridos de bananeira em quatro ciclos de produção. Pesquisa Agropecuária Brasileira, Brasília, v. 37, n. 11, p. 1.567-1.574, nov. 2002. 